\title{
Avaliação da atividade larvicida do óleo essencial do Zingiber officinale Roscoe (gengibre) frente ao mosquito Aedes aegypti
}

\author{
GOMES, P.R.B. ${ }^{*}$; SILVA, A.L.S.'; PINHEIRO, H.A. '; CARVALHO, L.L.'; LIMA, H.S.1; SILVA, E.F.'; SILVA, R.P.1; \\ LOUZEIRO, C.H.'; OLIVEIRA, M.B.'; FILHO, V.E.M.' \\ 'Universidade Federal do Maranhão, Departamento de Tecnologia Química, Laboratório de Pesquisa e Aplicação \\ de Óleo Essenciais, CEP 65080-805, São Luís, MA, Brasil. *Autor para correspondencia: prbgomes@yahoo. \\ com.br
}

RESUMO: Este trabalho analisa o efeito larvicida do óleo Zingiber officinale Roscoe contra larvas em terceiro estágio do mosquito Aedes aegypti. Extraiu-se quantitativamente o óleo essencial por hidrodestilação e calculou-se a $\mathrm{CL}_{50}$ do óleo, a partir dos métodos de Reed-Muench e Pizzi, respectivamente. O óleo essencial obteve $\mathrm{CL}_{50}$ de $76,07( \pm 2,24) \mu \mathrm{g} \mathrm{mL}-1$ e rendimento de $0,52 \%$ $\mathrm{m} / \mathrm{v}$. Os resultados indicam que o óleo essencial avaliado é composto por substâncias que propiciam efeito larvicida contra Aedes aegypti.

Palavras-chave: Óleo essencial, Zingiber officinale Roscoe, Atividade larvicida, Aedes aegypti.

\begin{abstract}
Evaluation of the larvicidal effectof the essential oil of Zingiber officinale Roscoe (ginger) against the mosquito Aedes aegypti. This study analyzes the larvicidal effect of the oil of Zingiber officinale Roscoe against larvae in third stage of the mosquito Aedes aegypti (Linnaeus 1792). The essential oil was extracted quantitatively by hydrodistillation and we calculated the oil's LC Lf $_{50}$ from Reed-Muench (1938) and Pizzi (1950) methods, respectively. The essential oil obtained $\mathrm{LC}_{50}$ of $76.07( \pm 2.24)$ ug $\mathrm{mL}^{-1}$, and yield of $0.52 \% \mathrm{~m} / \mathrm{v}$. The results indicate that the essential oil assessed has substances that provide larvicidal effect against Aedes aegypti.
\end{abstract}

Keywords: Essential oil, Zingiber officinale Roscoe, Larvicidal activity, Aedes aegypti.

\section{INTRODUÇÃO}

A dengue é uma doença de grande relevância para a medicina, pois é transmitida pelo mosquito Aedes aegypti, vetor de quatro sorotipos do flavivírus causador da dengue clássico e da febre hemorrágica. Portanto, torna-se necessário que o controle da densidade populacional do Aedes aegypti ocorra para que a dengue não assuma proporções de uma epidemia, pois não existe vacina para a doença, e a melhor forma de combatê-la é atacar o vetor, principalmente eliminando os locais onde ocorre a oviposição e o desenvolvimento das larvas do mosquito (Brasil, 2009).

Atualmente o controle é feito por meio de aplicações de inseticidas organofosforados e piretróides (Brasil, 2009). Porém, o uso frequente e em doses cada vez maiores desses produtos tem evidenciado os principais problemas devido ao uso destes inseticidas: o aparecimento de populações resistentes de mosquitos a esses produtos e os danos ambientais provocados por seu uso intensivo (Luna et al., 2004; Carvalho \& Silva, 2000; Polanczyk et al., 2003).
Por mais de 30 anos o organofosforado temephos foi o larvicida exclusivo usado no Brasil para o controle do Aedes aegypti (Barreto, 2005). Contudo, está sendo paulatinamente substituído; e a razão é o desenvolvimento de grande resistência das larvas, que não mais estavam sendo adequadamente controladas por aquele produto químico.

Uma alternativa tem sido o uso de plantas, as quais possuem substâncias cujas moléculas possuem ação fagoinibidora, repelente, inseticida, além de serem capazes de alterar a regulação do crescimento. Os óleos essenciais, produzidos no metabolismo secundário das plantas, também têm se apresentado como fontes de materiais com atividade inseticida, larvicida e repelente (Costa et al., 2005; Murugan et al., 2007).

Nos últimos 30 anos, têm sido aconselhado pela Organização Mundial de Saúde (OMS, 2011) os estudos com extratos de plantas consideradas medicinais (pelo conhecimento popular). A partir de 1978, segundo Soares (2002), a instituição passou a aconselhar a comunidade científica a intensificar 
e divulgar os estudos com plantas.

Entre os produtos obtidos de plantas, encontram-se os óleos essenciais de algumas espécies de plantas aromáticas, os quais já são largamente utilizados na indústria para a produção de sabonetes, perfumes e outros produtos de higiene pessoal. Estudos sobre a avaliação das atividades inseticida, bactericida e fungicida dos óleos essenciais de algumas espécies de plantas têm mostrado resultados interessantes, em várias pesquisas pelo mundo todo, e em especial em alguns grupos de pesquisa no Brasil (Lemos et al., 1990).

Nesse contexto, a procura por compostos larvicidas advindos de espécies vegetais, como os óleos essenciais extraídos de certas plantas, tem se intensificado.

Esses extratos de plantas, chamados "óleos essenciais", são misturas químicas complexas formadas, às vezes, por mais de cem componentes responsáveis, entre outras coisas, pelo seu aroma. Diferentes partes das plantas têm sido usadas para obtenção do óleo essencial: flores, folhas, sementes, raízes, frutos, cascas, rizomas e tubérculos (Aridogan et al., 2002).

Para o Brasil, pesquisas nesta área têm grande significado. No país, a dengue causa morte de muitas pessoas todos os anos ou a incapacidade de trabalho por um período de aproximadamente 10 dias devido aos sintomas geralmente severos. Isto representa uma perda de milhões de reais, tanto no investimento para erradicação da doença no Brasil, como na contagem dos dias de trabalho parado (Silva, 2006).

Tendo em vista o crescimento da doença e os prejuízos causados pela dengue no país, torna-se de suma importância a descoberta de novos materiais e desenvolvimento de métodos de combate ao seu vetor. Dessa forma, pelo fato de muitas plantas, por natureza, serem tóxicas para os mosquitos, os óleos essenciais podem representar uma saída eficiente para esse problema (Teles, 2003).

Nesse contexto, na busca por produtos naturais que possam ser usados como larvicidas contra Aedes aegypti no controle da transmissão da dengue, bem como seu uso potencial, foi testado o óleo essencial extraído dos rizomas de uma planta da flora brasileira, o Zingiber officinale Roscoe, contra as larvas desse mosquito.

\section{MATERIAL E MÉTODO}

O presente trabalho foi desenvolvido no Laboratório de Físico-Química do Pavilhão Tecnológico, Núcleo de Imunologia Básica e Aplicada
(NIBA) da Universidade Federal do Maranhão (UFMA), Central Analítica da Universidade de Campinas, Central Analítica da UFMA, Núcleo de Combustível, Catálise e Ambiental (NCCA).

Realizou-se o teste de atividade larvicida calculando-se a $\mathrm{CL}_{50}$ segundo o método ReedMuench (Colegate \& Molyneux, 1993).

\section{Obtenção do Óleo Essencial}

Os rizomas de gengibre foram obtidos em supermercado da rede varejista de São Luís, identificado como Zingiber officinale Roscoe, registrado no Herbário Ático Seabra (SLS) da Universidade Federal do Maranhão (UFMA) sob o número de registro 1125 e levado ao laboratório de Físico-Química de Alimentos do Pavilhão Tecnológico da Universidade Federal do Maranhão (UFMA), para extração do óleo essencial.

Para a extração do óleo essencial do Zingiber Officinale Roscoe utilizou-se um extrator de Clevenger de vidro acoplado a balão de fundo redondo de $6000 \mathrm{~mL}$ acondicionado em manta elétrica como fonte geradora de calor. A cada rotina de extração do óleo essencial, foram pesadas $400 \mathrm{~g}$ dos rizomas e a seguir triturados com água destilada na proporção 1:10 e colocadas em um balão de fundo redondo acoplado ao sistema extrator. A hidrodestilação foi conduzida a $100 \mathrm{oC}$ por 3,5 horas recolhendo-se o óleo essencial extraído. O óleo foi seco por meio de percolação em sulfato de sódio anidro $\left(\mathrm{Na}_{2} \mathrm{SO}_{4}\right)$. Essas operações foram realizadas em triplicatas e as amostras armazenadas em ampolas de vidro âmbar sob refrigeração de $4^{\circ} \mathrm{C}$ para evitar possíveis perdas de constituintes voláteis. Posteriormente esses óleos foram submetidos às análises.

O rendimento do óleo essencial foi expresso em porcentagem na relação massa/volume pela medida de densidade. Para realização dessa medida, foi utilizado um picnômetro de $1,0 \mathrm{~mL}$, previamente seco, tarado e aferido, onde se adicionaram as amostras a $25^{\circ} \mathrm{C}$, pesando-as em seguida. Após essa etapa, observou-se o volume $(\mathrm{mL})$ de óleo essencial obtido após a extração do óleo por massa (g) de material vegetal, conforme a fórmula descrito pela quarta edição da Farmacopeia Brasileira (1996) e por Fabrowski (2002).

\section{Obtenção e Cultivo das Larvas}

Como os ovos do Aedes aegypti não são postos diretamente na água, mas sim milímetros acima de sua superfície, principalmente em recipientes artificiais, foi preparada uma armadilha simples para coleta desses ovos. Para tanto, foram utilizados jarros de plástico para planta, de aproximadamente $500 \mathrm{~mL}$, semipreenchidos com água e um pedaço de madeira de dimensões de

Rev. Bras. PI. Med., Campinas, v.18, n.2, supl. I, p.597-604, 2016. 
aproximadamente $20 \mathrm{~cm} \times 5 \mathrm{~cm}$ com uma parte imersa e outra não. A fêmea do Aedes aegypti deposita seus ovos na parte imediatamente superior à lâmina d'água, na parte da madeira ainda úmida, mas fora da água do jarro.

Os ovos do Aedes aegypti foram imersos numa bacia plástica, de formato retangular, com cerca de 3 litros de água mineral para a eclosão. Após a imersão dos ovos, $0,5 \mathrm{~g}$ de ração de rato foi adicionado à água para auxiliar no crescimento das larvas. Todo o material foi mantido no interior de uma gaiola de madeira e coberta com uma tela de tecido, apropriada para insetos, a fim de evitar a contaminação por ovos de outras espécies de mosquito. Após a eclosão, as larvas foram acompanhadas até que atingissem o terceiro estágio do desenvolvimento, quando então foram utilizadas nos ensaios de atividade larvicida. Foram necessários de quatro a cinco dias para que as larvas atingissem o tamanho ideal para os ensaios.

As larvas foram identificadas como Aedes aegypti por técnicos do Laboratório do Núcleo de Patologia Tropical e Medicina Social do Departamento de Patologia da Universidade Federal do Maranhão.

\section{Análises Químicas}

Para as análises químicas, utilizou-se a técnica cromatografia em fase gasosa acoplada ao espectrômetro de massas por impacto de elétrons e analisador íon trap (CG-EM-IE-Ion trap). O equipamento utilizado foi da marca Varian 2100, utilizando hélio como gás de arraste com fluxo na coluna de $1 \mathrm{~mL} \mathrm{~min}-1$; temperatura do injetor de $270^{\circ} \mathrm{C}$, split 1:50; coluna capilar $(15 \mathrm{mx} 0,25 \mathrm{~mm})$ com fase

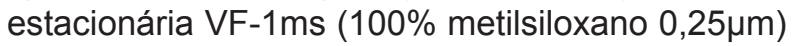
e programação de temperatura do forno de 60 a $200^{\circ} \mathrm{C}$ com taxa de aquecimento de $8^{\circ} \mathrm{C} \mathrm{min}$ me $^{-1}$, e de 200 a $290^{\circ} \mathrm{C}$ com taxa de aquecimento de $15^{\circ} \mathrm{C}$ min $^{-1}$. No Espectrômetro de Massas as temperaturas do mainfold, ion trap e da linha de transferência foram de $50^{\circ} \mathrm{C}, 190^{\circ} \mathrm{C}$ e $200^{\circ} \mathrm{C}$, respectivamente. Foram injetadas alíquotas de 1,0 $\mu \mathrm{L}$ (injetor automático CP-8410) das amostras diluídas na proporção de $20 \mu \mathrm{L}$ em $1,5 \mathrm{~mL}$ de hexano.

\section{Teste de Toxicidade}

Para a realização do teste de toxicidade, as larvas selecionadas no terceiro estágio foram transferidas para um béquer contendo $20 \mathrm{~mL}$ de água mineral com temperatura de 26 a $28^{\circ} \mathrm{C}$. Dez larvas foram capturadas utilizando-se pipeta Pasteur. Cada teste foi feito em quintuplicata para cada concentração testada. Os controles positivos foram realizados com o organofosforado temephos em larvas do Aedes aegypti, na concentração utilizada pela Vigilância Sanitária que é de 100 mg
$\mathrm{L}^{-1}$. Os controles negativos foram realizados com $20 \mathrm{~mL}$ de água mineral $\left(26-28^{\circ} \mathrm{C}\right)$ contendo $0,04 \%$ de Tween 80 (tensoativo hidrofólico que facilita a aplicação em emulsões do tipo óleo em água). As larvas foram expostas às soluções por 24 horas, sendo monitoradas de hora em hora. Ao fim dos períodos registrou-se a mortalidade.

Para o preparo da solução teste, pesou-se $20 \mathrm{mg}$ do óleo essencial em um recipiente (do tipo eppendorf), para cada mililitro da solução teste e, em seguida, foi adicionada uma gota de solvente do tipo Tween 80 sobre o óleo, fazendo-se então a homogeneização. A seguir, utilizando-se uma pipeta automática, foi adicionado um mililitro de água destilada fazendo-se nova homogeneização.

Esta solução foi então transferida para o béquer contendo as larvas separadas para o teste, de acordo com as concentrações pré-estabelecidas.

\section{Análise Estatística}

Após os testes, montou-se uma tabela com os valores das oito concentrações, logaritmo das mesmas, o número de larvas mortas após 24 horas (média dos cinco pontos), número de larvas vivas após 24 horas (média dos cinco pontos), o acumulado de mortos (soma das células de mortos abaixo) e o acumulado de vivos (soma das células de vivos acima).

A análise estatística dos dados foi realizada de acordo com o método de Reed \& Muench (1938), o qual parte do princípio de que um animal que sobreviva a certa dose, também irá sobreviver em qualquer outra dose menor que aquela, consequentemente, o animal que morrer com certa dose, também irá morrer em doses maiores que aquela. A partir de uma tabela contendo os dados de mortalidade para cada concentração testada, é construído um gráfico onde se observa uma curva para o acúmulo de animais mortos em cada concentração e outra curva para o acúmulo de sobreviventes. O ponto de intercessão entre as curvas é a Concentração Letal $50 \%\left(\mathrm{CL}_{50}\right)$, pois nesse ponto o número de animais sobreviventes é igual ao número de animais mortos (Colegate \& Molyneux, 1993).

O intervalo de confiança foi calculado segundo o método de Pizzi (1950) no qual se constrói um gráfico do percentual de mortos versus logaritmo (log) da dose. A seguir determina-se o valor de "R", que é a diferença entre o log da dose que mata $75 \%$ das larvas e o log da dose que mata $25 \%$ das larvas. Calcula-se também a variável "h" que consiste na média das diferenças dos valores de log das doses. Com esses dados determinase o log do erro padrão (SE), através da seguinte fórmula: $(S E)^{2}=0,79 \times h \times R / 20$. Finalmente, o valor do intervalo de confiança é igual $2 \times 10^{\text {sE }}$.

Rev. Bras. PI. Med., Campinas, v.18, n.2, supl. I, p.597-604, 2016. 


\section{RESULTADOS E DISCUSSÃO}

\section{Avaliação da cinética de extração e rendimento dos óleos essenciais}

A determinação das condições de extração de óleos voláteis é uma etapa muito importante por ser um fator determinante na relação entre a composição química e a qualidade do óleo extraído.

Segundo Mouchrek Filho (2000), o tempo de extração do óleo essencial é um dos principais parâmetros físico-químicos da indústria de essências, no que se refere à qualidade e à natureza econômica. Por isso, uma destilação rápida pode conduzir a um produto contendo predominantemente constituintes mais voláteis, porém destituído das melhores características; ao contrário, uma extração prolongada encarece o produto e também pode sobrecarregá-lo de compostos de aromas indesejáveis (Chaar, 2000).

O trabalho de extração do óleo essencial estudado foi realizado realizado em 5 horas, para a massa de $400 \mathrm{~g} / a m o s t r a$ e volume de 4 litros a $100^{\circ} \mathrm{C}$. Pode-se observar o tempo máximo de extração dos óleos na Figura 1.

A Figura 1 mostra que os tempos máximos de rendimento dos óleos essenciais em estudo e dos volumes obtidos, com relação ao tempo do rizoma de Zingiber officinale Roscoe foi de 3,5 horas, para o volume de $0,25 \mathrm{~mL}$.

O rendimento da extração foi calculado diante da quantidade de óleo que se obteve a partir de uma determinada massa do vegetal. Neste experimento partiu-se de $400 \mathrm{~g}$ de rizomas do Zingiber officinale Roscoe sendo obtido em média $0,25 \mathrm{~mL}$ de óleo. O rendimento massa/volume de óleo foi de $0,52 \%$ com densidade de 0,8280.

Ao extrair o óleo essencial do rizoma do gengibre, Martins (2010), obteve o rendimento de $0,37 \%$ em 4 horas, Tripathi et al. (2008) obtiveram $0,8 \%$ e Maia et al. (1991) em 7 horas obtiveram

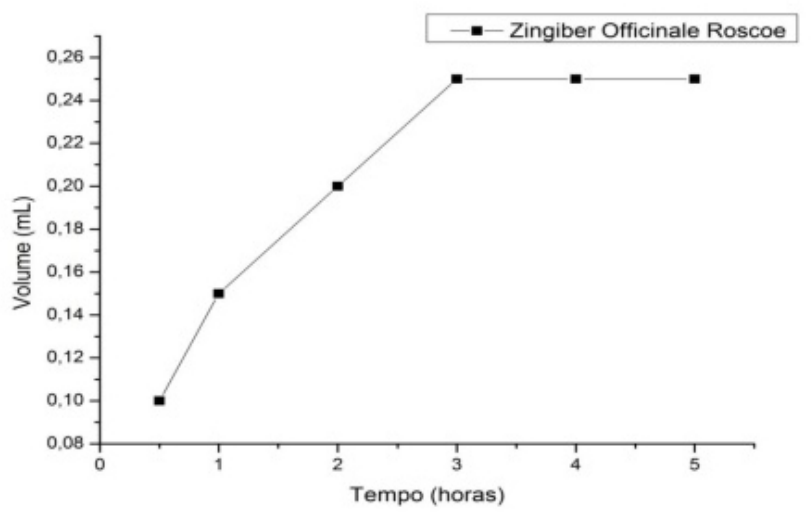

FIGURA 1. Cinética referente ao rendimento de extração do óleo em função do tempo, com massa de $400 \mathrm{~g}$ e temperatura de $100^{\circ} \mathrm{C}$. valores entre 0,56 e $0,92 \%$. Os volumes extraídos do óleo essencial estudado em relação aos da literatura estão bem próximos.

Conforme Özcan \& Chalchat (2002), a variação sazonal e a localidade são fatores importantes para diferentes variedades de plantas com relação aos rendimentos de extração encontrados na literatura.

Avaliação das características químicas dos óleos essenciais obtidas por Cromatografia Gasosa acoplada à Espectroscopia de Massas (CG-EM)

O resultado desse estudo nos mostra a presença de constituinte majoritário. O constituinte majoritário do óleo foi o a-zingibereno (sesquiterpeno) com $27,14 \%$, seguido pelo geranial (monoterpeno oxigenado) com $14,06 \%$, nerolidol (sesquiterpeno) com 13,51\%, neral (monoterpeno oxigenado) com 9,64\%, sesquifelandreno com $9,45 \%$, sabineno (monoterpeno hidrocarbono) com $5,23 \%$, canfeno (monoterpeno hidrocarbono) com $5,02 \%, 1,8$-cineol (monoterpeno oxigenado) com $4,35 \%$, ar-curcumeno (sesquiterpeno) com $3,33 \%$, a-pineno (monoterpeno) com $1,46 \%$, do $\beta$-mirceno (monoterpeno) com 1,29\%, farnesol com $1,27 \%$, nerol (monoterpeno) com 1,07\%, 4,4-dimetil-2pentinal com $0,80 \%$, terc-dodeciltiol com $0,71 \%$, 2-undecanona com 0,63\%, 1,1-diciclopropiletileno com $0,55 \%$ e linalol (monoterpeno) com $0,50 \%$.

Os resultados encontrados na presente pesquisa quanto à composição química do óleo essencial do gengibre revelaram que os mesmos foram semelhantes aos obtidos por Onyenekwe \& Hashimoto (1999) que ao analisarem a composição do óleo essencial do gengibre nigeriano constataram que o $\alpha$-zingibereno foi o componente majoritário com $29,54 \%$ do total do óleo. Em contra partida, o estudos realizados por Yu et al, (2007) empregando a técnica cromatografia gasosa acoplada a espectrometria de massa mostrou que os componentes majoritários do óleo essencial do Zingiber officinale Roscoe foram o $\beta$-felandreno e $\alpha$-zingibereno com percentuais respectivamente de 22,84 e 15,48.

\section{Atividade Larvicida}

A atividade larvicida do óleo essencial extraído dos rizomas do Zingiber officinale Roscoe foi testada em oito concentrações diferentes: $20,50,70,100,120,140,150$ e $160 \mu \mathrm{gL}^{-1}$, sendo $n$, o número de larvas do mosquito Aedes aegypti utilizadas no ensaio larvicida para cada concentração (totalizando 10 larvas por ensaio).

Para cada concentração os testes foram realizados em triplicata. Os dados sobre o número de larvas vivas e de larvas mortas foram

Rev. Bras. PI. Med., Campinas, v.18, n.2, supl. I, p.597-604, 2016. 
TABELA 1. Mortalidade das larvas do mosquito Aedes aegypti após 24 horas de exposição em várias concentrações do óleo essencial extraído dos rizomas do Zingiber officinale Roscoe.

\begin{tabular}{ccccccc}
\hline $\begin{array}{c}\text { Dose } \boldsymbol{\mu g} \\
\mathbf{m L}^{-1}\end{array}$ & Log dose & Mortos & Vivos & $\begin{array}{c}\text { Acumulados } \\
\text { mortos }\end{array}$ & $\begin{array}{c}\text { Acumulados } \\
\text { vivos }\end{array}$ & Mortalidade(\%) \\
\hline $\mathbf{1 6 0}$ & 2,2041 & 10 & 0 & 53 & 0 & 100 \\
$\mathbf{1 5 0}$ & 2,1761 & 9 & 1 & 43 & 1 & 90 \\
$\mathbf{1 4 0}$ & 2,1461 & 9 & 1 & 34 & 2 & 90 \\
$\mathbf{1 2 0}$ & 2,0792 & 8 & 2 & 25 & 4 & 80 \\
$\mathbf{1 0 0}$ & 2,0000 & 8 & 2 & 17 & 6 & 80 \\
$\mathbf{7 0}$ & 1,8451 & 5 & 5 & 9 & 11 & 50 \\
$\mathbf{5 0}$ & 1,6990 & 3 & 7 & 4 & 18 & 30 \\
$\mathbf{2 0}$ & 1,3010 & 1 & 9 & 1 & 27 & 10 \\
\hline
\end{tabular}

Número de larvas $(n=10)$

encontrados através da média de três repetições para cada uma das oito concentrações testadas (Tabela 1).

De acordo com a Tabela 1, a concentração de $20 \mu \mathrm{g} \mathrm{mL} \mathrm{m}^{-1}$ do óleo essencial dos rizomas do Zingiber officinale Roscoe apresentou a menor atividade larvicida, matando, em média, uma larva, o que corresponde a $10 \%$ de mortalidade. $\mathrm{Na}$ concentração de $70 \mu \mathrm{g} \mathrm{mL}^{-1}$ apresentou uma mortalidade de $50 \%$. A partir da concentração de $100 \mu \mathrm{g} \mathrm{mL}^{-1}$ do óleo essencial a atividade larvicida começou a crescer exponencialmente, até provocar a morte de $100 \%$ dos indivíduos testados com a concentração de $160 \mu \mathrm{g} \mathrm{mL}^{-1}$, ou seja, 10 larvas.

A taxa de percentagem da mortalidade das larvas frente o óleo é mostrada na Figura 2, mostrando-se o crescimento exponencial a partir do Log dose 2,0000 (concentração $100 \mu \mathrm{g} \mathrm{mL}-1$ ).

A Figura 3 mostra que a Concentração Letal $50 \%\left(\mathrm{CL}_{50}\right)$, concentração na qual cinquenta por cento das larvas testadas morrem, foi

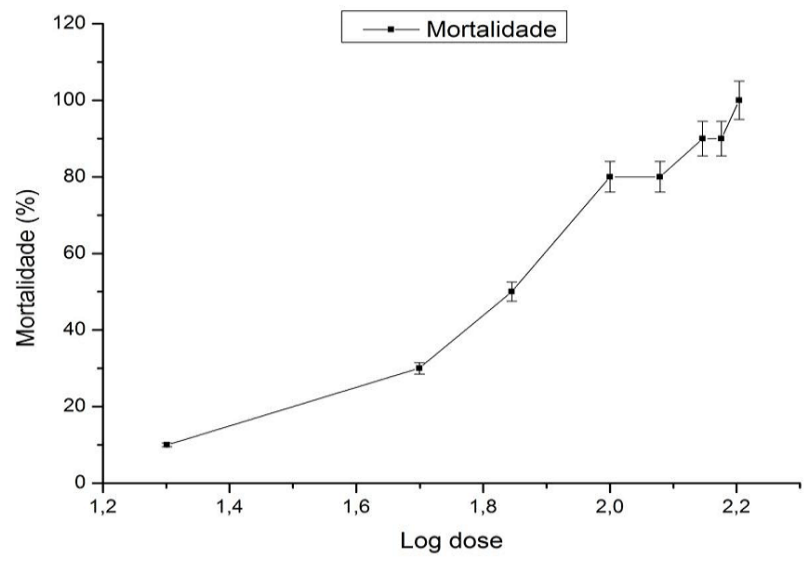

FIGURA 2. Taxa de mortalidade das larvas do mosquito Aedes aegypti - expostas a oito concentrações diferentes do óleo essencial dos rizomas do Zingiber officinale Roscoe, após 24 horas - versus o logaritmo de cada dose aplicada. encontrada próxima ao Log dose 1, 8689 (abaixo da concentração de $80 \mu \mathrm{g} \mathrm{mL}-1)$. De acordo com Cheng et al., (2003) valores de $\mathrm{CL}_{50}<100 \mu \mathrm{g} \mathrm{mL}^{-1}$ são considerados ativos.

A dose letal $50 \%$ para o óleo essencial dos rizomas do Zingiber officinale Roscoe foi calculada através da intersecção das curvas de indivíduos acumulados mortos e indivíduos acumulados vivos da Figura 3, tendo como resultado a concentração de $76,07 \mu \mathrm{g} \mathrm{mL}^{-1}$ com um intervalo de confiança de $2,24 \mu \mathrm{g} \mathrm{mL}^{-1}$.

A Concentração Letal $\left(\mathrm{CL}_{50}\right)$ obtida qualifica o óleo essencial como um agente larvicida em potencial (Cheng, et. al., 2003), que considera bons agentes larvicidas as substâncias com valor de CL50 inferior a $100 \mu \mathrm{g} \mathrm{mL-1}$.

A esse respeito, a potencialidade dos óleos essenciais varia segundo a espécie vegetal, a origem, a composição e os mecanismos de ação

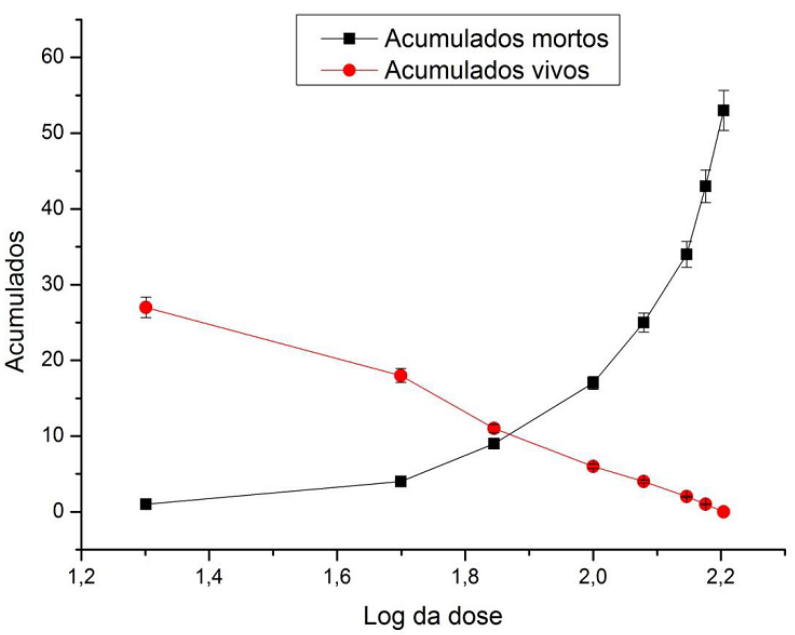

FIGURA 3. Estimativa da $\mathrm{CL}_{50}$ do óleo essencial dos rizomas do Zingiber officinale Roscoe pelo método Reed-Muench a partir do acumulado de larvas mortas e vivas em função do logaritmo decimal da dose aplicada. $A \mathrm{CL}_{50}$ é o ponto de intersecção das duas curvas. 
contra a praga em questão (Leyva, 2009; Teles, 2009).

Contra o Aedes aegypti alguns estudos mostram que os componentes terpênicos, alcoóis e aldeídos dos óleos essenciais são os principais responsáveis pela atividade inseticida ou larvicida (Lucia et al., 2007; Lee, 2006; Panella et al., 2005; Carvalho et al., 2003; Araújo et al., 2003; Karr \& Cotas, 1998).

É importante ressaltar, nesse contexto, que não se pode apenas atribuir a ação larvicida descrita neste estudo, portanto, ao composto a-Zingibereno particularmente; apesar de estar presente em maior quantidade, na ordem de $27 \%$, nos rizomas da amostra de gengibre.

Essa premissa é fortuitamente alegada quando nos reportamos a diversos achados na literatura referentes a alguns dos compostos identificados pelas técnicas analíticas utilizadas neste trabalho e que, possivelmente, podem ter contribuído na ação larvicida do óleo essencial dos rizomas do gengibre.

Santos et al. (2006) reportaram a atividade larvicida de mono e sesquisterpenos extraídos de óleos essenciais da família Boraginaceae frente ao Aedes aegypti. Entre os compostos majoritários os monoterpenos $\alpha$ - e $\beta$-pineno foram destacados como responsáveis pela ação larvicida (no presente estudo o primeiro composto apresentou um teor de 1,46\%).

Simas et al. (2004) pesquisando produtos naturais no controle do dengue identificaram diversas classes de terpenos, como os compostos $\alpha$ - pineno, $\beta$-pineno, linalol e eugenol. Os referidos autores indicam também que algumas características estruturais químicas são importantes para a atividade biológica, como por exemplo, a indicação de que a ligação dupla exocíclica no $\beta$-pineno parece ser mais importante que a ligação dupla endocíclica do a-pineno.

Os óleos essenciais podem atuar também em enzimas digestivas e neurológicas bem como interagir com o tegumento do inseto (Isman, 2006). Kim et al. (2003) demonstraram a importância da relação entre a estrutura química e atividade biológica dos compostos; reportando que quanto maior a lipofilicidade, maior a penetração no tegumento do inseto.

Outra observação de Simas et al. (2004) é quanto à importância da lipofilicidade de terpenos para a atividade larvicida em Aedes aegypti, quando se compararam monoterpenos e sesquiterpenos de estruturas correlatas.

Furtado et al. (2005) reportaram em seu estudo a ação larvicida de óleos contendo limoneno, linalol e eugenol frente ao mosquito Aedes aegypti. $\mathrm{Na}$ ocasião esses compostos foram confirmados como majoritários e apresentaram menores atividades quando testados isoladamente. Os autores também sugerem a possibilidade de outros compostos serem responsáveis pela atividade larvicida do óleo essencial ou ainda a existência de sinergismo entre o composto majoritário e outro(s) componente(s) do óleo, existentes em menor fração.

Como já relatado neste estudo, a melhor forma de combater a doença é erradicar o mosquito através do uso de larvicidas em seus locais de reprodução, por exemplo, o temephos que vem sendo utilizado pela Vigilância Sanitária. Entretanto, as larvas e os mosquitos já estão adquirindo resistência aos inseticidas sintéticos. Considera-se que os óleos essenciais de muitas plantas são por natureza tóxicos para os mosquitos, e menos agressores ao meio ambiente.

Em comparação ao principal larvicida utilizado no combate ao dengue, o temephos, estudos recentes quanto à susceptibilidade deste composto referem que se trata de um produto que com o passar dos anos perdeu a sua força frente ao mosquito, sendo necessárias doses cada vez maiores no seu combate (Furtado et al., 2005).

Prophiro (2008) estudou os efeitos do temephos sobre larvas do Aedes aegypti no terceiro estágio e verificou um processo de estabelecimento de resistência ao organofosforado em algumas cidades da região sul do Brasil.

Nesse contexto é válido relatar outra importante observação quanto à ineficiência crescente do pesticida temephos utilizado no Brasil pelo Programa Nacional de Controle da Dengue (PNCD): ele tem aspectos diferenciados no consumo do produto - enquanto na região Nordeste são consumidos $87,5 \mathrm{~g}$, no Sudeste o consumo é de $54,0 \mathrm{~g}$ e no Sul $48,0 \mathrm{~g}$ do produto (Augusto \& Camara Neto, 2007).

Diante desse panorama, os resultados alcançados com o óleo dos rizomas do Zingiber officinale Roscoe mostram a importância da necessidade de novas alternativas usando produtos de origem natural, especialmente se levarmos em conta que estes podem minimizar a dependência aos inseticidas químicos sintéticos.

Partindo dos resultados alcançados e do princípio de que o óleo essencial dos rizomas do Zingiber officinale Roscoe é um produto natural e, portanto, menos nocivo à saúde das pessoas e dos animais domésticos (Isman, 2000), podese afirmar que o óleo essencial é um promissor agente larvicida natural em locais de crescimento de larvas do Aedes aegypti.

Rev. Bras. PI. Med., Campinas, v.18, n.2, supl. I, p.597-604, 2016. 


\section{REFERÊNCIAS}

ARAÚJO, E. C. et al. Insecticidal activity and chemical composition of volatile oils from Hyptis martisii Benth. Journal of Agricultural and Food Chemistry, n. 51, p. 3260-2, 2003.

ARIDOGAN, B. C. et al., Antimicrobial activity and chemical composition of some essential oils. Archives of Pharmacal Research, v. 25, n. 6, p. 860-864, 2002.

AUGUSTO, L. G da S.; CÂMARA-NETO, H. F. Dengue: insustentabilidade do PEAa. In: XXVII CONGRESSO INTERAMERICANO DE ENGENHARIA SANITÁRIA E AMBIENTAL, 2007, Porto alegre-RS. Anais do XXVII Congresso Interamericano de Engenharia Sanitária e Ambiental. Porto Alegre: ABES, 2007.p.1-6.

BARRETO, C. F. Aedes aegypti - resistência aos inseticidas químicos e as novas alternativas de controle. Revista Eletrônica Faculdade Montes Belos, v. 1, n. 2, p. 62-73. 2005.

BRASIL. Ministério da Saúde. Vigilância Epidemiológica. Programa Nacional de Controle da Dengue. 2009.

CARVALHO, A. F. et al. Larvicidal activity of the essential oil from Lippia sidoide against Aedes aegypti. Memórias do Instituto Oswaldo Cruz, n. 98, p. 569-71, 2003.

CARVALHO, L. A. F.; SILVA, I. G. Avaliação longitudinal da atividade do Temephos a $1 \%$ sobre o Aedes aegypti (Lin., 1762). Entomologia y vectores, n. 7, p. 191-201, 2000.

CHAAR, J. S. Estudos analíticos e modificação química por acetilação do linalol contido no óleo essencial da espécie Aniba duckei Kostermans. 2000. 150 p. Tese (Doutorado em Química) - Programa de PósGraduação em Química, UFSCar, São Carlos.

CHENG, S.S. et al. Bioactivity of selected plant essential oils against the yellow fever mosquito Aedes aegypti larvae. Bioresource. Technology, v. 89, p.99-102, 2003.

COLEGATE, S. M. \& MOLYNEUX, R. J. Bioactive Natural Products: Detection, Isolation, and Structural Determination. Boca Raton: CRC Press, 1993.544 p.

COSTA, J. G. M. et al. Estudo químico-biológico dos óleos essenciais de Hyptis martiusii, Lippia sidoides e Syzigium aromaticum frente às larvas do Aedes aegypti. Revista Brasileira de Farmacognosia, v. 15, n. 4, p. 304-309, 2005.

FABROWSKI, F. J. R. T. BAKER (Myrtaceae). Eucaliptus smithii como espécie produtora de óleo essencial no sul do Brasil. 2002, 225 f. Tese (Doutorado em Engenharia Florestal) - Centro de Ciências Agrárias, Universidade Federal do Paraná.

FARMACOPEIA BRASILEIRA IV - PARTE 1. 4. ed. São Paulo: Editora Atheneu, 1996, 1.320p.

FURTADO, R. F. et al. Atividade Larvicida de Óleos Essenciais Contra Aedes aegypti L. (Diptera: Culicidae). Neotropical Entomology, v. 34, n. 5, p. 843-847, 2005.

ISMAN, M. B. Botanical insecticides, deterrents, and repellents in modern agriculture and an increasingly regulated world. Annual Review of Entomology, $n$. 51, p. 45-66, 2006.

ISMAN, M. Plant essential oil for pest and disease management. Crop Prot, n. 19, p. 603-08, 2000.

KARR, L.L.; COTAS, J.R. Insecticidal properties of dlimonelene. Journal of Pest Science, n. 3, p. 287-
90, 1998.

KIM, B. N. et al. Bacteremia due to Tribe Proteeae: a review of 132 cases during a decade (1991-2000). Scandinavian Journal of Infections Diseases. v. 35, n. 2, p. 98-103, 2003.

LEE, H. S. Mosquito larvicidal activity of aromatic medicinal planta oils against Aedes aegypti and Culex pipiens pallens. Journal of the American Mosquito Control Association, n. 22, p. 292-295, 2006.

LEMOS, T. L. G. et al. Antimicrobial activity of essential oils of Brazilian plants. Phytotherapy Research, v. 4, n. 2, p. 82-84, 1990.

LEYVA, M. et al. Actividad larvicida de aceites esenciales de plantas contra Aedes aegypti (L.) (Diptera: Culicidae). Revista Biomédica, n. 20, p. 5-13, 2009.

LUCIA, A. et al. Larvicidal effect of Eucalyptus grandis essential oil and turpentine and their major components on Ae. aegypti larvae. Journal of the American Mosquito Control Association, n. 23, p. 293-303, 2007.

LUNA, J. E. D. et al. Susceptibilidade de Aedes aegypti aos inseticidas temephos e cipermetrina, Brasil. Revista de Saúde Pública, n. 38, p. 842-843, 2004.

MAIA, N. B. et al. Obtenção e análise do óleo essencial do gengibre: efeito de secagem e processamento. Bragantia, v. 50, n. 1, p. 83-92, 1991.

MARTINS, A. G. L. A. Atividade antibacteriana dos óleos essenciais do manjericão (Ocimum basilicum Linnaeus) e do gengibre (Zingiber officinale Roscoe) frente a linhagens de Escherichia coli enteropatogênicas isoladas de hortaliças. 2010. 179 p. (Doutorado em Química) - Programa de PósGraduação em Química, PPGQ, UFPB, João Pessoa.

MOUCHREK FILHO, V.E. Estudos Analíticos e modificações químicas por metilação e acetilação do eugenol contido no óleo essencial extraído das folhas da espécie Pimenta dioica Lindl. 2000. 124p. Tese (Doutorado em Química) - Programa de PósGraduação em Química, UFSCar, São Carlos.

MURUGAN, K. et al. Larvicidal and repellent potential of Albizzia amara Boivin and Ocimum basilicum Linn against dengue vector, Aedes aegypti (Insecta:Diptera:Culicidae). Bioresource Technology, v.98, p. 198-201, 2007.

OMS. Organização Mundial de Saúde. OMS apóia estudo com plantas medicinais. Disponível em: <http:www. drashi.../fitoterapia_oms_apoia_estudos_com_plantas_ medicinais.ht> Acesso em 17 jul. 2011

ONYENEKWE, P.C.; HASHIMOTO, S. The composition of the essential oil of dried Nigerian ginger (Zingiber officinale Roscoe). European Journal Food Research and Technology, v.1999; n. 209, p. 407-410.

ÖZCAN, M.; CHALCHAT, J.C. Essential oil composition of Ocimum basilicum L. and Ocimum minimum L. in Turkey. Czech Journal of Food Sciences, v. 20, n. 6, p. 223-228, 2002.

PANELLA, N.A. et al. Use of novel compounds for pest control: insecticidal and acaricidal activity of essential oils components from heartwood of Alaska yellow cedar. Journal of Medical Entomology, n. 42, p. 352-358, 2005.

PIZZI, M. Sampling variation of the fifty percent end-point, determined by the Reed-Muench (Behrens) Method.

Rev. Bras. PI. Med., Campinas, v.18, n.2, supl. I, p.597-604, 2016. 
Human Biology, n. 22, p. 151-190, 1950.

POLANCZYK, R.A. et al. Potencial de Bacillus thuringiensis Berliner no controle de Aedes aegypti. Revista de Saúde Pública, n. 37, p. 813-816, 2003.

PROPHIRO, J. S. Susceptibilidade de Aedes aegypti (Linnaeus, 1762) e de Aedes albopictus (Skuse, 1894) (Diptera: Culicidae) a organofosforado e atividade inseticida de produtos de origem botânica. 2008. 121p. Dissertação (Mestrado em Ciências Biológicas) -, Programa de Pós-Graduação em Ciências Biológicas, UFPR, Curitiba.

REED L.J. \& MUENCH H. 1938. A simple method of estimating fifty percent endpoints.American Journal of Hygiene, v. 27, n. 3, p. 493-497, 1938.

SANTOS, R. P. et al. Chemical Composition and Larvicidal Activity of the Essential Oils of Cordia leucomalloides and Cordia curassavica from the Northeast of Brazil. Journal Brazilian Chemical Society, v. 17, n. 5, p. 1027-1030, 2006

SILVA, W. J. Atividade larvicida do óleo essencial de plantas existentes no Estado de Sergipe contra Aedes aegypti Linn. 2006. 81p. Dissertação (Mestrado em Desenvolvimento e Meio Ambiente) - Progama de Pós-Graduação em Desenvolvimento e Meio Ambiente, UFSE, São Cristóvão-SE.

SIMAS, N. K. et al. Produtos naturais para o controle da transmissão da dengue: atividade larvicida de Myroxylon balsamum (óleo vermelho) e de terpenóides e fenilterpenóides. Química Nova, v. 27, n. 01, p. 4649, fev. 2004.

SOARES, A. C. Se bem não fizer, mal também não fará. Revista Eletrônica de Ciências, São Carlos, n. 12, out. 2002. Disponível em:http://www.cdcc.sc.usp.br/ ciencia/artigos/art_12/medicamento.html> Acesso em 5 jan. 2011

TELES, R. M. Estudo analítico do linalol contido no óleo essencial extraído de galhos da espécie Aniba duckei Kostermans e sua aplicação como agente bactericida. 2003. 99p. Dissertação (Mestrado em Química) -Programa de Pós-Graduação em Química, UFMA, São Luís.

TRIPATHI, P. et al. Use of the essential oils as post-harvest botanical fungicides in the management of grey mould of grapes caused by Botrytis cinerea, World Journal of Microbiology and Biotechnology, v. 24, n. 1, p. 39-46, 2008.

YU, Y. et al. Development of gas chromatographymass spectrometry with microwave distillation and simultaneous solid-phase microextraction for rapid determination of volatile constituents in ginger. Journal of Pharmaceutical and Biomedical Analysis. v. 43, n. 1, p. 24-31, 2007. 